\title{
Wogonin reduces cardiomyocyte apoptosis from mitochondrial release of cytochrome $c$ to improve doxorubicin-induced cardiotoxicity
}

\author{
YUNJIE WEI $^{1 *}$, JUNHAO ZHAO $^{2 *}$, JIAN XIONG $^{1}$, JINGJING CHAI $^{3}$, XI YANG $^{1}$, \\ JUNFENG WANG $^{1}$, JIAJUAN CHEN ${ }^{1}$ and JING WANG \\ ${ }^{1}$ Department of Cardiology, Taihe Hospital, Hubei University of Medicine, Shiyan, Hubei 442000; \\ ${ }^{2}$ The First Clinical College, Jinyun Mountain Campus of Chongqing Medical University, Chongqing 401331; \\ Departments of ${ }^{3}$ Clinical Oncology and ${ }^{4}$ Neurology, Taihe Hospital, Hubei \\ University of Medicine, Shiyan, Hubei 442000, P.R. China
}

Received September 23, 2021; Accepted November 29, 2021

DOI: $10.3892 /$ etm.2022.11128

\begin{abstract}
Doxorubicin (DOX) has powerful anticancer properties, but its clinical application is affected by its serious cardiotoxicity. Wogonin (WG) has been shown to have marked cardiovascular protection potential. However, it is not known whether this potential can protect the heart from DOX damage. The aim of the present study was to investigate whether WG could ameliorate the cardiotoxicity of DOX. DOX and WG were used to establish a model of cardiac damage. Echocardiography, brain natriuretic peptide, creatine kinase $\mathrm{MB}$ and cardiac troponin $\mathrm{T}$ were used to detect the degree of cardiac damage. The levels of superoxide dismutase, malondialdehyde, glutathione and catalase in serum were measured to observed oxidative stress state. The mRNA levels of cyclophilin D, voltage-dependent anion-selective channel 1 and adenine nucleotide transporter 1 were detected by reverse transcription-quantitative PCR. Western blotting was used to detect the expression of cytochrome $c$ in mitochondria and cytoplasm and cleaved-caspase- 9 and pro/cleaved-caspase- 3 in cytoplasm in cardiac tissue and primary cardiomyocytes to verify the related signaling pathways. DOX rats showed a series of cardiac damage. However, these damages were alleviated following WG treatment. Further studies showed that WG antagonized DOX cardiotoxicity through inhibiting the release of cytochrome $c$. WG protected rat heart
\end{abstract}

Correspondence to: Dr Yunjie Wei, Department of Cardiology, Taihe Hospital, Hubei University of Medicine, 32 Renmin South Road, Shiyan, Hubei 442000, P.R. China

E-mail: weiyunjie200@163.com

${ }^{*}$ Contributed equally

Key words: wogonin, doxorubicin, cardiotoxicity, cytochrome $c$, anti-apoptotic from DOX damage. The mechanism may be closely related to inhibiting the release of cytochrome $c$ from mitochondria and reducing cardiomyocyte apoptosis caused by caspase activation.

\section{Introduction}

Doxorubicin (DOX) is a double-edged sword as a classic anthracycline anti-tumor drug (1). On one hand, it is favored by doctors and patients because of its powerful anti-tumor effects (2). On the other hand, its dose accumulation and time-dependent cardiotoxicity concerns doctors and patients (3). The mechanism of cardiac toxicity caused by DOX is mostly related to the increase of oxidative stress, damage to mitochondrial function and finally apoptosis (4). Cytochrome c serves a key role in mitochondrial injury (5). The increased permeability of damaged mitochondrial membrane leads to the leakage of cytochrome $c$ from mitochondria and finally leads to cardiomyocyte apoptosis (6). Although some drugs have been used in the prevention and treatment of DOX-induced cardiotoxicity for a number of years, the overall effect remains poor $(7,8)$.

At present, dexrazoxane is the only anthracycline anti-tumor drug cardioprotective agent approved by the FDA (USA), but it is rarely used clinically in China due to its high price and lack of popularity $(9,10)$. Therefore, it is urgent to find a more effective protective drug to protect the heart from the damage of anthracycline anti-tumor drugs.

Wogonin (WG; 5,7-dihydroxy-8-methoxyflavone), an important flavonoid extracted from Scutellaria baicalensis root, has been proved to possess anti-inflammatory, antioxidant, anti-allergic and anti-apoptotic properties $(11,12)$. A number of studies have shown that it protects the heart from diabetes, obesity and ischemia-reperfusion injury (13-15). However, whether WG can protect heart from DOX damage remains to be elucidated.

In the present study, DOX was used to induce cardiac injury and WG was administered by gavage to observe its protective effect. The aim of the present study was to investigate the 
anti-apoptotic effect of WG on DOX-induced cardiotoxicity and its key mechanism.

\section{Materials and methods}

Materials. Wogonin (WG) and Doxorubicin (DOX), purity $\geq 98 \%$, were purchased from MilliporeSigma. Malondialdehyde (MDA; cat. no. A003-1-1) and superoxide dismutase (SOD; cat. no. A001-1-1) test kits were obtained from Nanjing Jiancheng Bioengineering Institute. Brain natriuretic peptide (BNP; cat. no. 1608B), creatine kinase MB (CK-MB; cat. no. 6930B) and cardiac troponin $\mathrm{T}$ (cTnT; cat. no. 7278A) test kits were all purchased from Shanghai Meixuan Biotechnology Co., Ltd. ROS detection kit and CCK-8 cell viability and toxicity detection kit were purchased from Biosharp Life Sciences. The antibody of cytochrome $c$ (cat. no. 11940), COX IV (cat. no. 4850), pro/cleaved-caspase-3 (cat. no. 14220S/cat. no. 9664S) and cleaved-caspase-9 (cat. no. 9507) were obtained from Cell Signaling Technology, Inc. All chemicals and reagents were analytical grade.

Animal model. The present study was approved by the Animal Ethics Committee of Hubei Medical College (approval no. 42000900000093). A total of 40 Male SD rats (weight, 150-180 g; age, 5-6 weeks) were obtained from the Hubei Medical College experimental animal center. Rats were randomly distributed equally into four groups: CON group (normal, $\mathrm{n}=10$ ), WG group (WG gavage, $100 \mathrm{mg} \mathrm{kg}^{-1}, \mathrm{n}=10$ ), DOX group ( $3 \mathrm{mg} \mathrm{kg}^{-1}$ DOX was injected via caudal vein once a week, $\mathrm{n}=10)$ and WG+THP group $\left(3 \mathrm{mg} \mathrm{kg}^{-1}\right.$ THP was injected via caudal vein once a week with WG gavage, $100 \mathrm{mg}$ $\left.\mathrm{kg}^{-1}, \mathrm{n}=10\right)$. The rats were kept at a standard room temperature of $22 \pm 3^{\circ} \mathrm{C}, 45 \pm 10 \%$ humidity and 12 -h light/dark cycle. The animals were supplied with standard laboratory food and ad libitum access to tap water before experiments. The dosages of DOX and WG in animal experiments referred to relevant studies (16-18).

Electrocardiography and echocardiography. Following the experiments, rats were anesthetized by ketamine $(55 \mathrm{mg} / \mathrm{kg})$ plus xylazine (15 mg/kg) (19). Following anesthesia, Doppler echocardiography of rats were measured by a Vivid E95 ultrasonic diagnostic instrument (General Electric Company) and ejection fraction (EF) and fractional shortening (FS) values in echocardiography were measured. electrocardiograms (ECG) of rats were recorded by BL-420E biological function measurement system (Chengdu Taimeng Technology Co., Ltd.) and the R-wave, T-wave and QT interval in ECG were measured.

Sample collection and processing. Under anesthesia, the rats were sacrificed by cervical dislocation. Blood samples were collected and centrifuged at $3,000 \mathrm{xg}$ for $30 \mathrm{~min}$ at $4^{\circ} \mathrm{C}$ immediately. The supernatant was collected and the levels of BNP, CK-MB, cTnT, SOD and MDA were measured immediately according to the kit instructions. Part of the left ventricular tissue was fixed in 10 times volume of $4 \%$ paraformaldehyde solution for $72 \mathrm{~h}$ at $4^{\circ} \mathrm{C}$. Then, following dehydration and infiltration, it was embedded in paraffin and sectioned at 4-5 $\mu \mathrm{m}$ thickness and finally stained according to the instructions of H\&E and TUNEL staining kit (Beyotime Institute of Biotechnology) for $1 \mathrm{~h}$ at room temperature. The remaining heart tissue was cryopreserved at $-80^{\circ} \mathrm{C}$.

Cell culture and treatment. The ventricular parts of neonatal $\mathrm{SD}$ rats were quickly separated under sterile conditions, cleaned and then cut into fragments with a diameter of less than $1 \mathrm{~mm}$, digested by trypsin + type II collagenase, then filtered, centrifuged at $1,000 \mathrm{x}$ g for $5 \mathrm{~min}$ at room temperature, suspended and seeded $\left(\sim 5 \times 10^{6}\right.$ cells/well). Finally, primary rat cardiomyocytes were isolated by the differential adhesion method (5). The primary cardiomyocytes were divided into four groups: Normal group (CON), WG group (WG, $10 \mu \mathrm{M}$, $12 \mathrm{~h}$ ), DOX group (DOX, $5 \mu \mathrm{M}, 12 \mathrm{~h}$ ), DOX and WG co-culture group (DOX, $5 \mu \mathrm{M}+\mathrm{WG} 10 \mu \mathrm{M}, 12 \mathrm{~h}$ ).

Western blotting. The mitochondria and cytoplasm of rat ventricular tissue and primary rat cardiomyocytes were separated according to the instructions of tissue or cell mitochondrial extraction kit (Beyotime Institute of Biotechnology). A BCA kit was used to determine the protein concentration thereafter. Then, $\sim 30 \mu \mathrm{g}$ heart tissue or cell lysate was loaded per lane onto $12 \%$ sodium dodecyl sulfate-polyacrylamide gel electrophoresis, after which proteins were transferred to an FL membrane (MilliporeSigma) at $4^{\circ} \mathrm{C}$ for $2 \mathrm{~h}$. After blocking with 5\% blocking protein powder (non-fat milk powder; Beyotime Institute of Biotechnology) at room temperature for $2 \mathrm{~h}$, the following primary antibodies were added and incubated overnight at $4^{\circ} \mathrm{C}$ : Cyt $\mathrm{C}(1: 1,000)$, pro/cleaved caspase-3 (cat. no. $14220 \mathrm{~S} /$ cat. no. $9664 \mathrm{~S} ; 1: 1,000$ ), cleaved caspase-9 (cat. no. 9507; 1:1,000), COX IV (cat. no. 4850; 1:1,000) and GAPDH (cat. no. 10494-1-AP; 1:5,000). Subsequently, HRP-conjugated goat anti-rabbit IgG $(\mathrm{H}+\mathrm{L})$ secondary antibodies (1:10,000; Thermo Fisher Scientific, Inc.; cat. no. \#31460) were added and incubated at room temperature for $1.5 \mathrm{~h}$. The results of western blotting were analyzed using BeyoECL Plus (Beyotime Institute of Biotechnology) and Image Lab software (version 5.2.1; Bio-Rad Laboratories, Inc.). The specific protein expression levels were normalized to GAPDH or COX IV.

Reverse transcription-quantitative (RT-q) PCR. TRIzol ${ }^{\circledR}$ (Thermo Fisher Scientific, Inc.) was used to extract total RNA from frozen, crushed rat hearts and $5 \times 10^{6}$ cultured primary cardiac myocytes $(n=3)$ in accordance with the manufacturers protocol, and quantified at $260 \mathrm{~nm}$ using a NanoPhotometer (cat. no. P300; Implen GmbH). The total RNA was transcribed in two steps by super script first strand synthesis system used in accordance with the manufacturers protocol (cat. no. K1073; Apexbio Technology LLC). The PCR products were quantified by SYBR Green PCR Master Mix (Applied Biosystems; Thermo Fisher Scientific, Inc.) and the results were standardized with $\beta$-Actin Gene expression. The thermocycling conditions were as follows: Initial denaturation at $95^{\circ} \mathrm{C}$ for $30 \mathrm{sec}$, followed by 40 cycles of denaturation at $94^{\circ} \mathrm{C}$ for $5 \mathrm{sec}$, annealing at $58^{\circ} \mathrm{C}$ for $30 \mathrm{sec}$ and extension at $70^{\circ} \mathrm{C}$ for $5 \mathrm{sec}$. $\beta$-actin served as the internal reference gene and results were analyzed using the $2^{-\Delta \Delta \mathrm{Cq}}$ method (20). The primer sequences were: Voltage-dependent anion-selective channel 1 (VDAC-1): 
Forward ATGTCTTCACCAAGGGCTAT, Reverse TCT GGGTCACTCGGGATT; adenine nucleotide transporter 1 (ANT1): Forward TGGGCGACTGTATCATCAAG, Reverse TCACACTCTGGGCAATCATC; cyclophilin D (Cyp D): Forward TTCCATCTTATGCTCTTCACCG, Reverse GGT TGAAGAAGTCCTTGTCTGC; $\beta$-actin: Forward CTCTTC CAGCCTTCCTTCCT, Reverse AGCACTGTGTTGGCG TACAG.

Statistical analysis. Data were presented as mean $\pm \mathrm{SD}$. The significance of differences between groups were analyzed statistically using one way analysis of variance (ANOVA), followed by a Tukey's multiple-comparison post hoc test. $\mathrm{P}<0.05$ was considered to indicate a statistically significant difference.

\section{Results}

WG improves the abnormalities of echocardiography and $E C G$ in DOX rats. At the end of the experiment, a certain number of succumbed in DOX group and the mortality was $\sim 30 \%$ (7 survived and 3 succumbed). There was no mortality the in other groups.

Following DOX administration, echocardiography and ECG changes occurred in rats, suggesting that DOX caused cardiac injury in rats. Echocardiography (Fig. 1A) showed a decrease in EF (Fig. 1C) and FS (Fig. 1D) in rats. However, the changes of EF and FS were reversed following WG treatment. Similar to echocardiography, DOX also caused ECG (Fig. 1B) abnormalities in rats, which were characterized by increased $\mathrm{R}$ wave (Fig. 1E) and T wave (Fig. 1F) and prolonged QT interval (Fig. 1G). WG treatment also reversed these changes.

WG improves myocardial injury and increase of oxidative stress in DOX rats. Myocardial injury is often accompanied by the increase of serum myocardial injury markers and myocardial oxidative stress (21). In the DOX rat model, the contents of serum cTnT (Fig. 1H), CK-MB (Fig. 1I) and BNP (Fig. 1J) increased. However, the above myocardial injury markers decreased following WG treatment. Similarly, in myocardial tissue, SOD activity (Fig. 2A), glutathione (GSH) content (Fig. 2C) and CAT content (Fig. 2D) decreased and MDA content (Fig. 2B) increased. WG effectively alleviated the abnormal oxidative stress. These results suggested that WG effectively alleviated the heart injury caused by DOX and the abnormal increase of oxidative stress.

WG improves DOX induced cardiac tissue damage and cardiomyocyte apoptosis in rats. DOX caused the disorder of cardiac tissue arrangement, the increase of cell gap and the abnormality of nucleus in rats. The related changes were alleviated following WG treatment (Fig. 2F). As shown in Fig. 2E, TUNEL staining showed that there was no cardiomyocyte apoptosis in CON and WG group, but there was regional cardiomyocyte apoptosis in DOX injection group. The treatment of WG effectively improved DOX-induced cardiomyocyte apoptosis. The quantitative results were shown in Fig. 2G.

Effect of WG on DOX-induced apoptosis-related signal pathway in rats. Mitochondrial membranes are composed of
Cyp D, VDAC1 and ANT1. Abnormal increased permeability cause cytochrome $c$ to leak into cytoplasm from mitochondria and start caspase related apoptosis signal pathway. The mRNA level of the components of mitochondrial membranes was detected by RT-qPCR. The results showed that the mRNA expressions of Cyp D, VDAC1 and ANT1 in DOX model rats were significantly higher than those in the control group (Fig. 3A). As shown in Fig. 3A, the mRNA levels of these genes in the WG group were significantly lower than those in the DOX group, which showed that under the action of WG, there were fewer mitochondrial permeability conversion pores composed of the above components than DOX group, which inhibited the release of cytochrome $c$ from mitochondria to cytoplasm.

By contrast, western blotting was used to detect the changes of mitochondrial membrane permeability related apoptosis signal pathway. The results showed that the expression of mitochondrial cytochrome $c$ protein in DOX rats was significantly lower compared with the control group (Fig. 3B), while the expression of cytoplasmic cytochrome $c$ protein was significantly higher compared with that in the control group (Fig. 3D). This indicated that cytochrome $c$ leaked from mitochondria into the cytoplasm. In addition, the cytoplasmic cleaved-caspase-9/3 of DOX model rats was significantly higher compared with of the control group, while pro-caspase-3 was significantly lower compared with of the control group (Fig. 3D). This suggests that caspase dependent apoptosis was activated. However, compared with DOX group, WG treatment group effectively reversed the above changes (Fig. 3B and D). The semi-quantitative analysis results were shown in Fig. 3C and E.

Effect of WG on DOX-induced apoptosis-related signal pathway in cardiomyocytes. In order to verify whether WG has the same protective effect on DOX induced-cardiomyocyte apoptosis in cells, a DOX cell model we cultured and treated with WG. As with the animal experiments, WG prevented the abnormal increase of mitochondrial membrane permeability caused by DOX, the leakage of cytochrome $c$ from mitochondria into cytoplasm and the activation of caspase dependent apoptosis pathway in DOX cell group.

Specifically, WG effectively inhibited the significant increase of Cyp D, VDAC1 and ANT1 mRNA expression (Fig. 4A), the significant decrease of mitochondrial cytochrome $c$ (Fig. 4B) and cytoplasmic pro-caspase-3 protein expression (Fig. 4D) and the significant increase of cytoplasmic cytochrome $c$ and cleaved-caspase-9/3 (Fig. 4D) protein expression caused by DOX. The semi-quantitative analysis results were shown in Fig. 4C and E.

\section{Discussion}

Doxorubicin (DOX) is an anthracycline anti-tumor antibiotic, which has a wide spectrum of anti-tumor effects (2). It is commonly used in the treatment of acute leukemia, breast cancer, malignant lymphoma and bronchial lung cancer (22-24). However, its clinical application is seriously limited due to its dose-dependent and time-dependent cardiotoxicity $(3,7,25)$. The cardiotoxicity of DOX is often manifested as arrhythmia, myocardial conduction disorder, myocarditis, cardiomyopathy 
A

CON

WG

DOX

$\mathrm{DOX}+\mathrm{WG}$
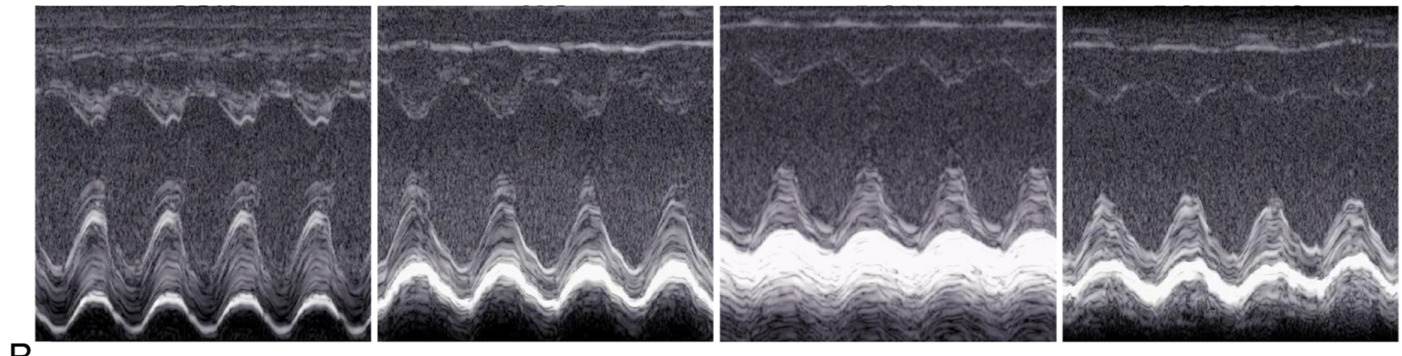

B
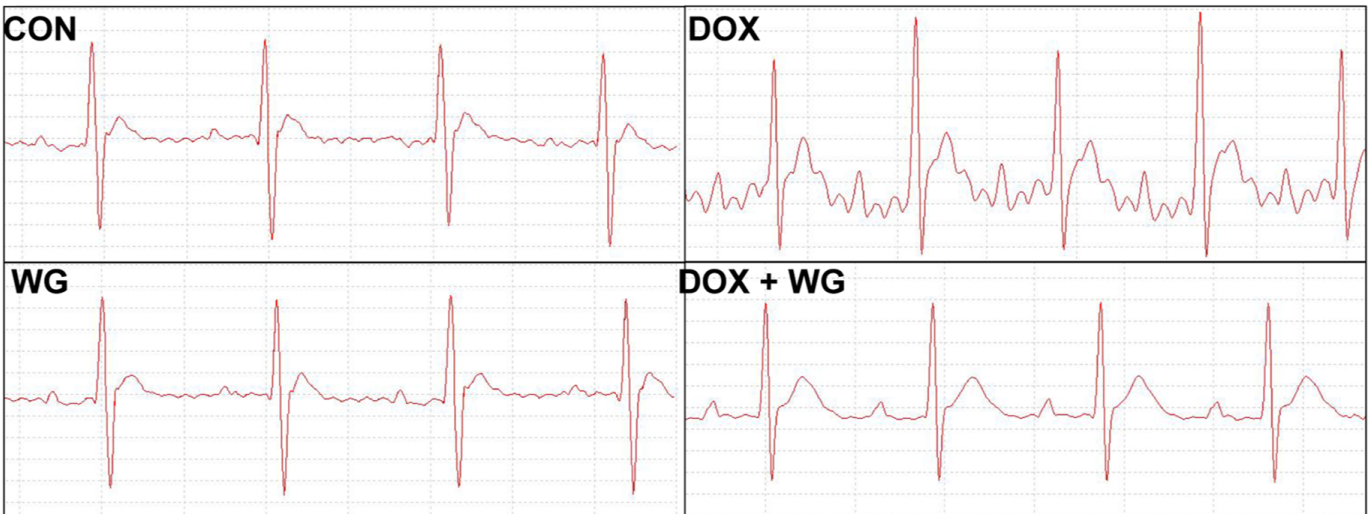

C

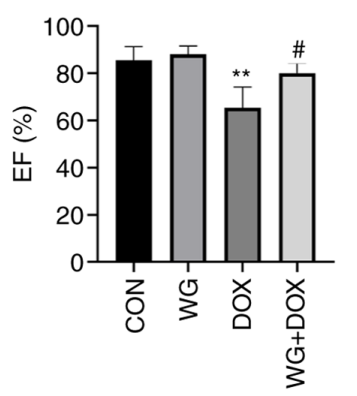

G

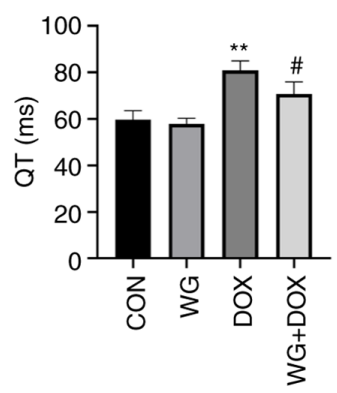

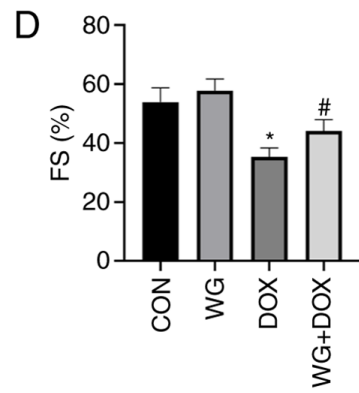
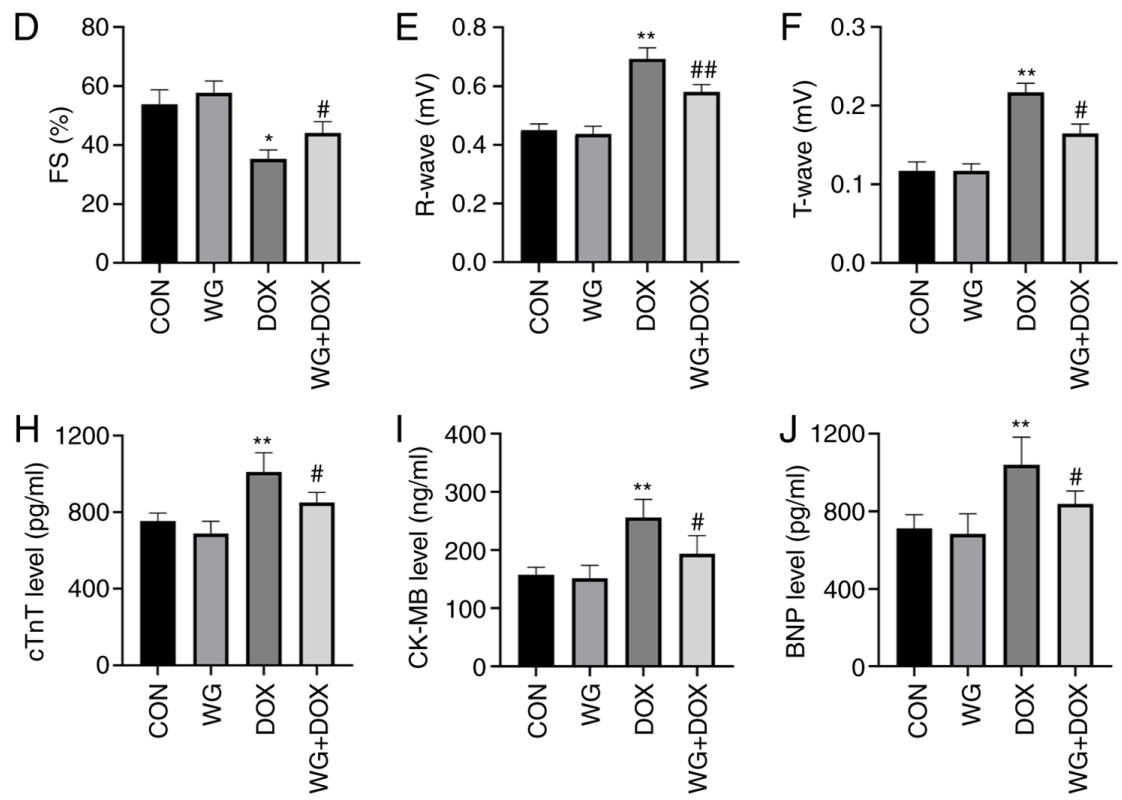

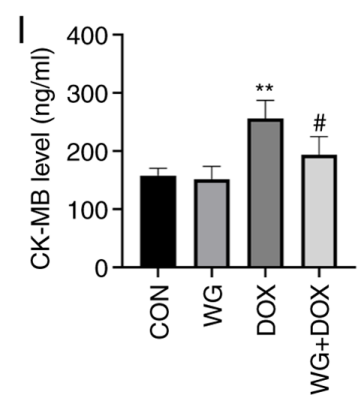

Figure 1. WG ameliorates DOX-induced myocardial injury in rats. WG improved the damage induced by DOX in rats as shown by of (A) echocardiography and (B) ECG. Specifically, WG reversed the decrease of (C) EF and (D) FS, the increase of (E) R wave and (F) T wave and the prolongation of (G) QT interval in DOX rats. In addition, WG also improved the contents of serum (H) cTnT, (I) CK-MB and (J) BNP in DOX rats. All values are the mean \pm SD. $P<0.05$, ${ }^{* *} \mathrm{P}<0.01$ vs. CON; ${ }^{\#} \mathrm{P}>0.05,{ }^{\# \#} \mathrm{P}>0.01$ vs. DOX. WG, wogonin; DOX, doxorubicin; ECG, electrocardiograms; EF, ejection fraction; FS, fractional shortening; CON, control; cTnT, cardiac troponin T; CK-MB, creatine kinase MB; BNP, brain natriuretic peptide.

and heart failure (26). Traditional wisdom suggests that the safe cumulative dose of DOX is $550 \mathrm{mg} / \mathrm{m}^{2}$ (27). However, there are obvious individual differences in the cardiotoxicity of DOX and there is no absolute safe dose (28). Previous studies have confirmed that cardiomyocyte apoptosis serves a very important role in DOX-induced cardiotoxicity $(29,30)$. DOX leads to a large amount of ROS accumulation and calcium overload in cells and destroys the permeability of mitochondrial membrane $(31,32)$. A large number of pro-apoptotic factors such as cytochrome $c$ enter the cytoplasm from mitochondria, activate the caspase pathway, initiate the endogenous apoptosis pathway and finally lead to cardiomyocyte death (33). Other studies also found that DOX can cause abnormal mitochondrial morphology and structure, lipid deposition and irregular arrangement of myofilaments, accompanied by myofilament breakage and dissolution $(4,10)$. This indicates that mitochondrial function serves an important role in DOX induced heart injury.

WG, also known as 5,7-dihydroxy-8-methoxyflavone, is a flavonoid extracted from the root of Scutellaria baicalensis. It 

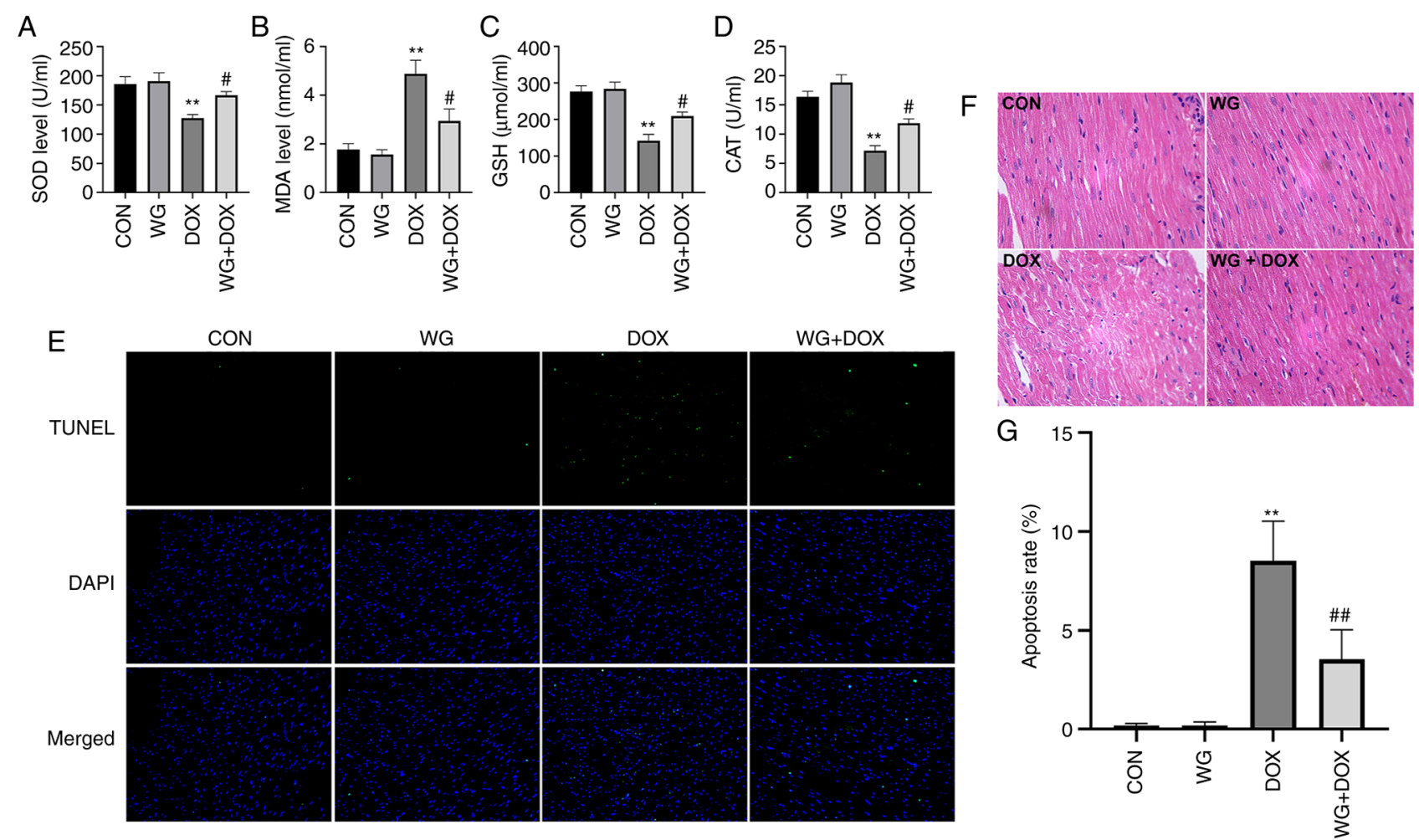

Figure 2. WG improves the DOX-induced oxidative stress and apoptosis in rat hearts. WG reversed the decrease of (A) SOD, (C) GSH and (D) CAT and the increase of (B) MDA in myocardial tissue of DOX rats. (E) WG also improved cardiomyocyte apoptosis in DOX rats (magnification, $\mathrm{x} 200)$. (F) Hematoxylin and eosin staining (magnification, $\mathrm{x} 200$ ). (G) Quantitative analysis. All values are the mean $\pm \mathrm{SD} .{ }^{* *} \mathrm{P}<0.01 \mathrm{vs}$. CON; ${ }^{*} \mathrm{P}>0.05$, ${ }^{* \#} \mathrm{P}>0.01 \mathrm{vs}$. DOX. WG, wogonin; DOX, doxorubicin; SOD, superoxide dismutase; GSH, glutathione; CAT, catalase; MDA, malondialdehyde.

A

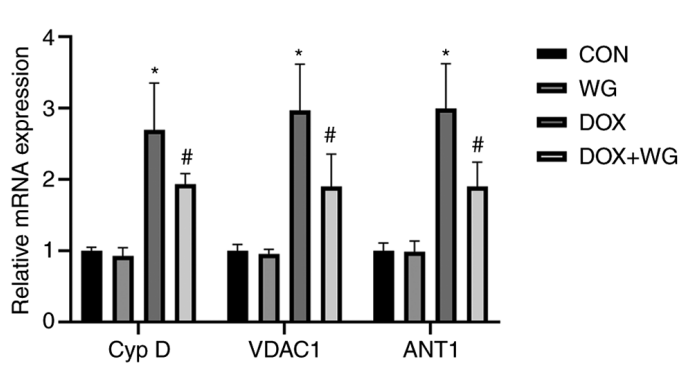

D

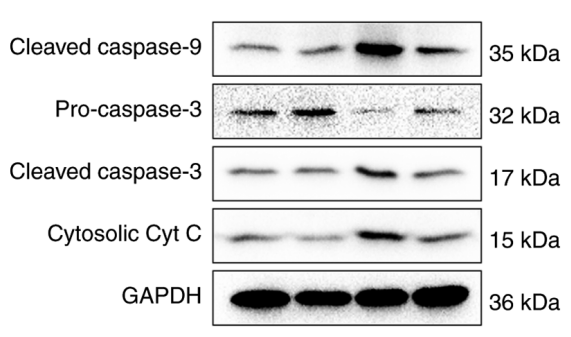

B

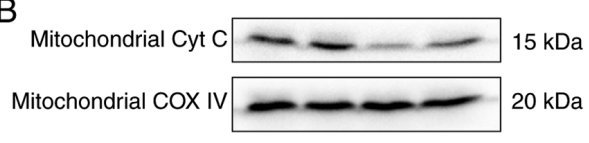

C
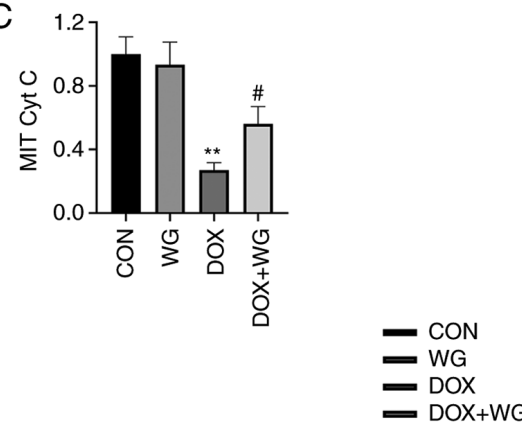

$\mathrm{E}$

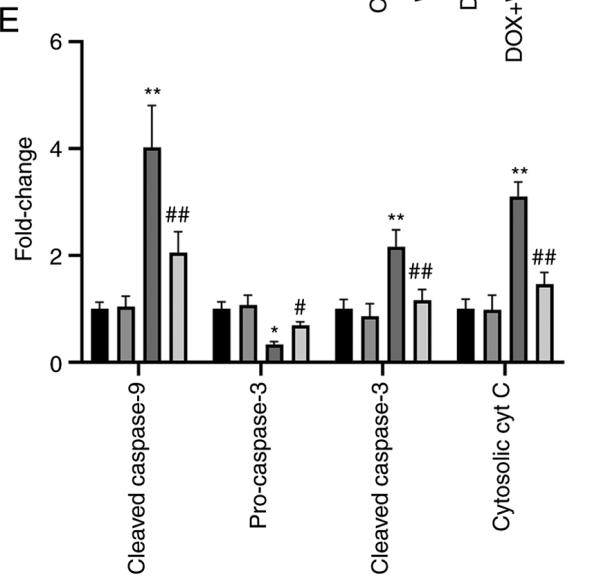

Figure 3. Effects of WG and DOX on apoptosis related signaling pathways in rat heart. (A) WG effectively improved the increased mRNA expression of Cyp D, VDAC1 and ANT1 in DOX rats, (B) the decreased expression of mitochondrial cytochrome $c$ protein and (D) the increased the expression of cytoplasmic cytochrome $c$ protein, the increased expression of cleaved-caspase-9/3 and the decreased expression of pro-caspase-3. (C and E) Semi quantitative analysis. The expression level of mitochondrial Cyt $\mathrm{C}$ specific protein was normalized to COX IV. All values are the mean $\pm \mathrm{SD}$. ${ }^{*} \mathrm{P}<0.05,{ }^{* *} \mathrm{P}<0.01$ vs. CON; ${ }^{\#} \mathrm{P}>0.05$, ${ }^{\# \# P}>0.01$ vs. DOX. WG, wogonin; DOX, doxorubicin; VDAC1, voltage-dependent anion-selective channel 1; Cyp D, cyclophilin D; ANT1, adenine nucleotide transporter 1; Cyt C, cytochrome $c$. 
A

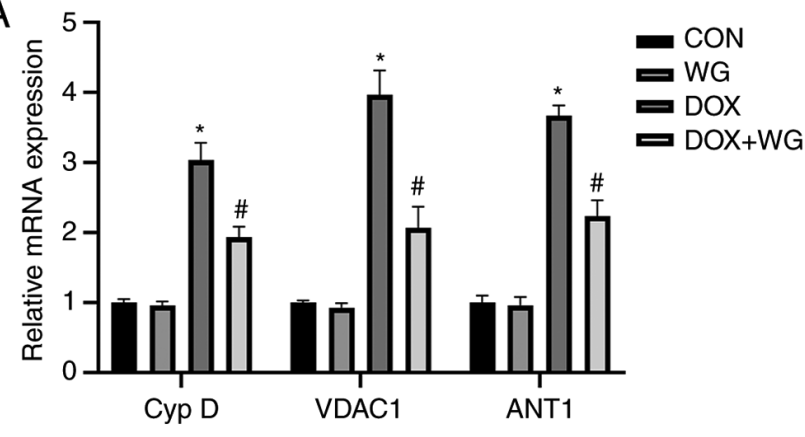

B

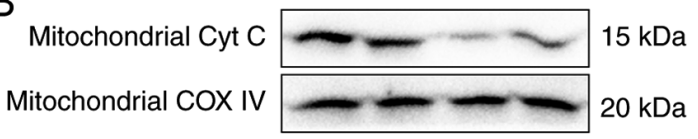

C

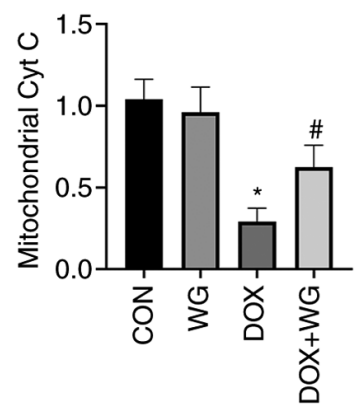

E

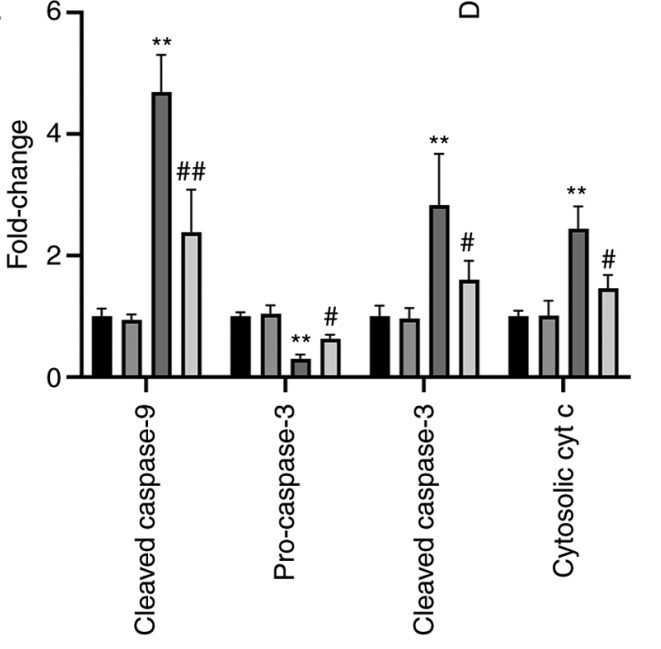

Figure 4. Effects of WG and DOX on apoptosis related signaling pathways in cardiomyocytes. (A) As in the animal models, WG effectively improved the increased mRNA expression of Cyp D, VDAC1 and ANT1 in DOX rats, (B) the decreased expression of mitochondrial cytochrome $c$ protein and (D) the increased the expression of cytoplasmic cytochrome $c$ protein and the increased expression of cleaved-caspase-9/3 and the decreased expression of pre-caspase-3. (C and E) semi-quantitative analysis. All values are the mean $\pm \mathrm{SD} .{ }^{*} \mathrm{P}<0.05,{ }^{* *} \mathrm{P}<0.01 \mathrm{vs}$. CON, ${ }^{\#} \mathrm{P}>0.05$, ${ }^{\# \#} \mathrm{P}>0.01 \mathrm{vs}$. DOX. WG, wogonin; $\mathrm{DOX}$, doxorubicin; VDAC1, voltage-dependent anion-selective channel 1; Cyp D, cyclophilin D; ANT1, adenine nucleotide transporter 1.

has significant pharmacological effects in a variety of diseases, such as anti-inflammatory, anti-apoptosis, anti-oxidation, cell cycle regulation and anti-cancer and leukemia $(13,15)$. In addition, WG also shows powerful cardioprotective effect in animal models $(14,15)$, but its pharmacological effect in heart injury caused by DOX remains to be elucidated. The present study showed that $100 \mathrm{mg} / \mathrm{kg}$ WG improved the changes of echocardiography and ECG induced by DOX and decreased the serum levels of BNP, CK-MB and cTnT (34). Elevated levels of cardiac markers suggested that DOX caused myocardial injury, but WG effectively alleviated the heart injury caused by DOX.

In the present study, it was also observed that the serum SOD, GSH and CAT increased and the serum MDA level decreased in DOX rats following WG treatment, indicating that WG exerts an anti-oxidant effect. Relevant studies have pointed out that WG significantly reduces the myocardial infarction area, serum cardiac markers, lipid peroxidation products (MDA) and inflammatory markers in myocardial infarction rats. Significantly upregulating the expression of Nrf2 and HO-1 protein has also been demonstrated, which has a powerful myocardial protective effect on myocardial injury $(11,13,15)$.

Compared with DOX group, WG decreased the levels of cytosolic cytochrome $c$ and cleaved-caspase-3/9 protein and increased mitochondrial cytochrome $c$. At the same time, WG also reduced the levels of VDAC1, ANT1 and Cyp D mRNA and the reduction of mitochondrial cytochrome $c$ release at the gene level, thus reducing the occurrence of apoptosis; this is similar to the results of Zhang et al (33) on the cardiotoxicity of another anthracycline in rats. Mitochondrial function and its role in maintaining cellular redox/oxidation balance are the basis of regulating cellular homeostasis (35). ROS is the main by-product of normal mitochondrial metabolism and stable internal environment (36). Under physiological conditions, the balance between ROS generation and clearance is highly controlled (37). DOX causes excessive accumulation of ROS and upregulates oxidation, which may lead to serious mitochondrial damage, cell damage and death and lead to the failure of the whole organ and organism $(38,39)$. Mitochondrial permeability transition pore (mPTP) serves a central role in regulating cell death (40). A number of factors, such as high concentration of ROS, seem to lead to the opening of mPTP (41). The mPTP is hypothesized to be regulated by VDAC in mitochondrial outer membrane, ANT in mitochondrial inner membrane and Cyp D in mitochondrial matrix $(40,42)$. The abnormal increase of mPTP permeability from various reasons leads to apoptosis through mitochondrial mediated pathway (43). In short, DOX significantly increases the level of cytochrome $c$ 
in cytoplasm and the expression of cleaved-caspase-3/9 protein and decreases the level of cytochrome $c$ in mitochondria. Cytochrome $\mathrm{c}$ is not only an important factor for mitochondria to promote apoptosis, but also a key factor for the initiation of apoptosis signal pathway $(44,45)$. Once released, cytochrome $c$ forms a complex with Apaf1 and pre-caspase-9, which activates cleaved-caspase-9/3, resulting in programmed cell death $(45,46)$. The opening of mPTP leads to mitochondrial depolarization and mitochondrial membrane potential dissipation, followed by progressive mitochondrial swelling and the loss of soluble components of respiratory chain, which eventually leads to the rupture of mitochondrial outer membrane and the leakage of proteins from mitochondria to cytoplasm, thus accelerating the occurrence of apoptosis $(47,48)$. The present study showed that WG reversed the opening of mPTP and reduced the release of apoptotic factors cytochrome $c$ into the cytoplasm, so as to prevent apoptosis. DOX can directly activate cardiomyocyte apoptosis by reducing Bcl-2/Bax ratio (49). Previous studies have noted that the high expression of $\mathrm{Bcl}-2$ can enhance the resistance of cells to most DNA damage factors and inhibit the apoptosis of target cells caused by most chemotherapeutic drugs and that the Bax gene, which inhibits $\mathrm{Bcl}-2$, is the main apoptosis gene in human body $(50,51)$. The proportional relationship between $\mathrm{Bax} / \mathrm{Bcl}-2$ protein is one of the key factors to determine the inhibitory effect on apoptosis $(5,52)$. However, whether WG also has the ability to change the increase of $\mathrm{Bax} / \mathrm{Bcl}-2$ ratio caused by DOX to resist cardiomyocyte apoptosis remains to be elucidated.

In conclusion, the present study showed that WG significantly reduced DOX-induced cardiac toxicity in rats. The anti-apoptotic activity may be partly responsible for the cardioprotective effect of WG. The present study reported that WG reduces the release of cytochrome $c$ by changing mitochondrial permeability and serve an anti-apoptotic role to achieve cardiac protection. However, more studies are required to determine the specific mechanism of WG's anti-apoptotic effect and promote the clinical application of WG in the future. The present study also lacked the corresponding positive drug control of mitochondrial protective agents and the specific difference between WG and traditional cardiovascular protective drugs is not clear; this will also be the further direction of research.

\section{Acknowledgements}

Not applicable.

\section{Funding}

No funding was received.

\section{Availability of data and materials}

The datasets used and/or analyzed during the current study are available from the corresponding author on reasonable request.

\section{Authors' contributions}

YW, JZ, JX, JCha, XY, JuW, JChe and JiW substantially contributed to the conception and the design of the study, and in the acquisition, analysis and interpretation of the data. JZ and JX contributed to manuscript drafting or critical revisions on intellectual content. YW and JZ approved the final manuscript version to be published. YW and JZ agreed to be accountable for all aspects of the work, so that any questions relating to research integrity or scientific accuracy in any part of the study are appropriately investigated and resolved. YW and JZ confirm the authenticity of all the raw data. All authors have read and approved the final manuscript.

\section{Ethics approval and consent to participate}

The present study was approved by the Animal Ethics Committee of Hubei Medical College.

\section{Patient consent for publication}

Not applicable.

\section{Competing interests}

The authors declare that they have no competing interests.

\section{References}

1. Speth PA, van Hoesel QG and Haanen C: Clinical pharmacokinetics of doxorubicin. Clin Pharmacokinet 15: 15-31, 1988.

2. Nam J, Son S, Ochyl LJ, Kuai R, Schwendeman A and Moon JJ: Chemo-photothermal therapy combination elicits anti-tumor immunity against advanced metastatic cancer. Nat Commun 9: 1074, 2018.

3. Koleini $\mathrm{N}$ and Kardami E: Autophagy and mitophagy in the context of doxorubicin-induced cardiotoxicity. Oncotarget 8: 46663-46680, 2017.

4. Tadokoro T, Ikeda M, Ide T, Deguchi H, Ikeda S, Okabe K, Ishikita A, Matsushima S, Koumura T, Yamada KI, et al: Mitochondria-dependent ferroptosis plays a pivotal role in doxorubicin cardiotoxicity. JCI Insight 5: e132747, 2020.

5. Shi H, Tang H, Ai W, Zeng Q, Yang H, Zhu F, Wei Y, Feng R, Wen L, Pu P and He Q: Schisandrin B antagonizes cardiotoxicity induced by pirarubicin by inhibiting mitochondrial permeability transition pore (mPTP) opening and decreasing cardiomyocyte apoptosis. Front Pharmacol 12: 733805, 2021

6. Han Y, Cai Y, Lai X, Wang Z, Wei S, Tan K, Xu M and Xie H: lncRNA RMRP prevents mitochondrial dysfunction and cardiomyocyte apoptosis via the miR-1-5p/hsp70 axis in LPS-induced sepsis mice. Inflammation 43: 605-618, 2020.

7. Songbo M, Lang H, Xinyong C, Bin X, Ping Z and Liang S: Oxidative stress injury in doxorubicin-induced cardiotoxicity. Toxicol Lett 307: 41-48, 2019.

8. Wenningmann N, Knapp M, Ande A, Vaidya TR and Ait-Oudhia S: Insights into doxorubicin-induced cardiotoxicity: Molecular mechanisms, preventive strategies, and early monitoring. Mol Pharmacol 96: 219-232, 2019.

9. Fang X, Wang H, Han D, Xie E, Yang X, Wei J, Gu S, Gao F, Zhu N, Yin X, et al: Ferroptosis as a target for protection against cardiomyopathy. Proc Natl Acad Sci USA 116: 2672-2680, 2019.

10. Sangweni NF, Moremane M, Riedel S, van Vuuren D, Huisamen B, Mabasa L, Barry R and Johnson R: The prophylactic effect of pinocembrin against doxorubicin-induced cardiotoxicity in an in vitro H9c2 cell model. Front Pharmacol 11: 1172, 2020.

11. Khan NM, Haseeb A, Ansari MY, Devarapalli P, Haynie S and Haqqi TM: Wogonin, a plant derived small molecule, exerts potent anti-inflammatory and chondroprotective effects through the activation of ROS/ERK/Nrf2 signaling pathways in human osteoarthritis chondrocytes. Free Radic Biol Med 106: 288-301, 2017.

12. Lucas CD, Dorward DA, Sharma S, Rennie J, Felton JM, Alessandri AL, Duffin R, Schwarze J, Haslett C and Rossi AG: Wogonin induces eosinophil apoptosis and attenuates allergic airway inflammation. Am J Respir Crit Care Med 191: 626-636, 2015. 
13. Shi X, Zhang B, Chu Z, Han B, Zhang X, Huang P and Han J: Wogonin inhibits cardiac hypertrophy by activating Nrf-2-mediated antioxidant responses. Cardiovasc Ther 2021: 9995342, 2021.

14. Chang WT, Shao ZH, Vanden Hoek TL, McEntee E, Mehendale SR, Li J, Becker LB and Yuan CS: Cardioprotective effects of grape seed proanthocyanidins, baicalin and wogonin: Comparison between acute and chronic treatments. Am J Chin Med 34: 363-365, 2006.

15. Bei W, Jing L and Chen N: Cardio protective role of wogonin loaded nanoparticle against isoproterenol induced myocardial infarction by moderating oxidative stress and inflammation. Colloids Surf B Biointerfaces 185: 110635, 2020.

16. Hydock DS, Lien CY, Jensen BT, Parry TL, Schneider CM and Hayward R: Rehabilitative exercise in a rat model of doxorubicin cardiotoxicity. Exp Biol Med (Maywood) 237: 1483-1492, 2012.

17. Hiensch AE, Bolam KA, Mijwel S, Jeneson JAL, Huitema ADR Kranenburg O, van der Wall E, Rundqvist H, Wengstrom Y and May AM: Doxorubicin-induced skeletal muscle atrophy: Elucidating the underlying molecular pathways. Acta Physiol (Oxf) 229: e13400, 2020.

18. Badawy AM, El-Naga RN, Gad AM, Tadros MG and Fawzy HM: Wogonin pre-treatment attenuates cisplatin-induced nephrotoxicity in rats: Impact on PPAR- $\gamma$, inflammation, apoptosis and Wnt/ $\beta$-catenin pathway. Chem Biol Interact 308: 137-146, 2019.

19. Luo S, Wang T, Qin H, Lei H and Xia Y: Obligatory role of heat shock protein 90 in iNOS induction. Am J Physiol Cell Physiol 301: C227-C233, 2011.

20. Livak KJ and Schmittgen TD: Analysis of relative gene expression data using real-time quantitative PCR and the 2(-Delta Delta C(T)) method. Methods 25: 402-408, 2001

21. Shi H, Zeng Q, Wei Y, Yang H, Tang H, Wang D, Pu P and Feng R Canagliflozin is a potential cardioprotective drug but exerts no significant effects on pirarubicin-induced cardiotoxicity in rats. Mol Med Rep 24: 703, 2021.

22. Zhang K, Guo QL, You QD, Yang Y, Zhang HW, Yang L, Gu HY, Qi Q, Tan Z and Wang X: Wogonin induces the granulocytic differentiation of human NB4 promyelocytic leukemia cells and up-regulates phospholipid scramblase 1 gene expression. Cancer Sci 99: 689-695, 2008.

23. Yang D, Guo Q, Liang Y, Zhao Y, Tian X, Ye Y, Tian J, Wu T and $\mathrm{Lu} \mathrm{N}$ : Wogonin induces cellular senescence in breast cancer via suppressing TXNRD2 expression. Arch Toxicol 94: 3433-3447, 2020.

24. Wang $C$ and Cui C: Inhibition of lung cancer proliferation by wogonin is associated with activation of apoptosis and generation of reactive oxygen species. Balkan Med J 37: 29-33, 2019.

25. Kalyanaraman B: Teaching the basics of the mechanism of doxorubicin-induced cardiotoxicity: Have we been barking up the wrong tree? Redox Biol 29: 101394, 2020.

26. Ruggeri C, Gioffré S, Achilli F, Colombo GI and D'Alessandra Y: Role of microRNAs in doxorubicin-induced cardiotoxicity: An overview of preclinical models and cancer patients. Heart Fai Rev 23: 109-122, 2018.

27. Swain SM, Whaley FS and Ewer MS: Congestive heart failure in patients treated with doxorubicin: A retrospective analysis of three trials. Cancer 97: 2869-2879, 2003.

28. Nagy L, Szabó F, Iványi J, Németh L, Kovács GL, Palatka J, Tarján J, Tóth K and Roth E: A method for detection of doxorubicin-induced cardiotoxicity: Flow-mediated vasodilation of the brachial artery. Exp Clin Cardiol 6: 87-92, 2001.

29. Zhang X, Hu C, Kong CY, Song P, Wu HM, Xu SC, Yuan YP, Deng W, Ma ZG and Tang QZ: FNDC5 alleviates oxidative stress and cardiomyocyte apoptosis in doxorubicin-induced cardiotoxicity via activating AKT. Cell Death Differ 27: 540-555, 2020.

30. Zheng X, Zhong T, Ma Y, Wan X, Qin A, Yao B, Zou H, Song Y and Yin D: Bnip3 mediates doxorubicin-induced cardiomyocyte pyroptosis via caspase-3/GSDME. Life Sci 242: 117186, 2020.

31. Minotti G, Menna P, Salvatorelli E, Cairo G and Gianni L: Anthracyclines: Molecular advances and pharmacologic developments in antitumor activity and cardiotoxicity. Pharmacol Rev 56: 185-229, 2004.
32. Yun $\mathrm{CH}$, Chae HJ, Kim HR and Ahn T: Doxorubicin- and daunorubicin-induced regulation of $\mathrm{Ca}^{2+}$ and $\mathrm{H}^{+}$fluxes through human bax inhibitor-1 reconstituted into membranes. J Pharm Sci 101: 1314-1326, 2012.

33. Zhang Y, Ma XY, Zhang T, Qin M, Sun B, Li Q, Hu DW and Ren LQ: Protective effects of apocynum venetum against pirarubicin-induced cardiotoxicity. Am J Chin Med 47: 1075-1097, 2019.

34. Irfan A, Reichlin T, TwerenboldR,Fischer C, Ballarino P,Nelles B, Wildi K, Zellweger C, Rubini Gimenez M, Mueller M, et al: Cardiomyocyte injury induced by hemodynamic cardiac stress: Differential release of cardiac biomarkers. Clin Biochem 48: $1225-1229,2015$

35. Lee CF, Chavez JD, Garcia-Menendez L, Choi Y, Roe ND, Chiao YA, Edgar JS, Goo YA, Goodlett DR, Bruce JE and Tian R: Normalization of NAD ${ }^{+}$redox balance as a therapy for heart failure. Circulation 134: 883-894, 2016.

36. Mazat JP, Devin A and Ransac S: Modelling mitochondrial ROS production by the respiratory chain. Cell Mol Life Sci 77: 455-465, 2020

37. Poljsak B, Suput D and Milisav I: Achieving the balance between ROS and antioxidants: When to use the synthetic antioxidants. Oxid Med Cell Longev 2013: 956792, 2013

38. Zorov DB, Juhaszova M and Sollott SJ: Mitochondrial reactive oxygen species (ROS) and ROS-induced ROS release. Physiol Rev 94: 909-950, 2014.

39. Bartlett JJ, Trivedi PC and Pulinilkunnil T: Autophagic dysregulation in doxorubicin cardiomyopathy. J Mol Cell Cardiol 104: $1-8,2017$.

40. Bauer TM and Murphy E: Role of mitochondrial calcium and the permeability transition pore in regulating cell death. Circ Res 126: 280-293, 2020.

41. Rottenberg H and Hoek JB: The path from mitochondrial ROS to aging runs through the mitochondrial permeability transition pore. Aging Cell 16: 943-955, 2017.

42. Halestrap AP: What is the mitochondrial permeability transition pore? J Mol Cell Cardiol 46: 821-831, 2009.

43. Baechler BL, Bloemberg D and Quadrilatero J: Mitophagy regulates mitochondrial network signaling, oxidative stress, and apoptosis during myoblast differentiation. Autophagy 15: 1606-1619, 2019

44. Ow YP, Green DR, Hao Z and Mak TW: Cytochrome c: Functions beyond respiration. Nat Rev Mol Cell Biol 9: 532-542, 2008.

45. Bock FJ and Tait SWG: Mitochondria as multifaceted regulators of cell death. Nat Rev Mol Cell Biol 21: 85-100, 2020.

46. Jiang X and Wang X: Cytochrome C-mediated apoptosis. Annu Rev Biochem 73: 87-106, 2004.

47. Kwong JQ and Molkentin JD: Physiological and pathological roles of the mitochondrial permeability transition pore in the heart. Cell Metab 21: 206-214, 2015.

48. Javadov S and Karmazyn M: Mitochondrial permeability transition pore opening as an endpoint to initiate cell death and as a putative target for cardioprotection. Cell Physiol Biochem 20: $1-22,2007$

49. Chang WT, Lin YW, Ho CH, Chen ZC, Liu PY and Shih JY: Dapagliflozin suppresses ER stress and protects doxorubicin-induced cardiotoxicity in breast cancer patients. Arch Toxicol 95: 659-671, 2021.

50. Ashkenazi A, Fairbrother WJ, Leverson JD and Souers AJ: From basic apoptosis discoveries to advanced selective BCL-2 family inhibitors. Nat Rev Drug Discov 16: 273-284, 2017.

51. Adams JM and Cory S: The Bcl-2 protein family: Arbiters of cell survival. Science 281: 1322-1326, 1998.

52. Song S, Chu L, Liang H, Chen J, Liang J, Huang Z, Zhang B and Chen X: Protective effects of dioscin against doxorubicin-induced hepatotoxicity via regulation of Sirt1/FOXO1/NF-kb signal. Front Pharmacol 10: 1030, 2019.

This work is licensed under a Creative Commons Attribution-NonCommercial-NoDerivatives 4.0 International (CC BY-NC-ND 4.0) License. 